\title{
Lifetime effects in color superconductivity at weak coupling
}

\author{
Cristina Manuel* \\ Theory Division, CERN, CH-1211 Geneva 23, Switzerland
}

(Received 27 June 2000; published 25 October 2000)

\begin{abstract}
Present computations of the gap of color superconductivity in weak coupling assume that the quarks which participate in the condensation process are infinitely long lived. However, the quasiparticles in a plasma are characterized by having a finite lifetime. In this article we take into account this fact to evaluate its effect in the computation of the color gap. By first considering the Schwinger-Dyson equations in weak coupling, when one-loop self-energy corrections are included, a general gap equation is written in terms of the spectral densities of the quasiparticles. To evaluate lifetime effects, we then model the spectral density by a Lorentzian function. We argue that the decay of the quasiparticles limits their efficiency to condense. The value of the gap at the Fermi surface is then reduced. To leading order, these lifetime effects can be taken into account by replacing the coupling constant of the gap equation by a reduced effective one.
\end{abstract}

PACS number(s): 12.38.Mh, 24.85.+p

\section{INTRODUCTION}

In QCD at high baryonic density, when asymptotic freedom implies that the interactions are weak, matter behaves as a color superconductor [1-3]. This is a consequence of Cooper's theorem, as any attractive interaction occurring close to the Fermi surface makes the system unstable to the formation of particle pairing. In QCD the attractive interaction is provided by one-gluon exchange between quarks in a color antisymmetric $\overline{3}$ channel.

In the weak coupling regime it is possible to compute the value of the quark-quark condensate [4-9]. The condensation process is dominated by the exchange of very soft magnetic gluons [4], which are dynamically screened by medium effects. A careful analysis of the gap equation, as arising from the Schwinger-Dyson equations, was done in Refs. [5-7], and the value of the gap was determined up to a constant of order 1 . The inclusion of Meissner effects in the gluon exchange processes introduces a slight correction to the value of the gap [10]. The gap can also be computed directly from the scattering matrix, looking into the instability in the proper vertex function $[11,12]$. From this approach, the undetermined constant of the gap was computed.

In this article we study the gap equation when the oneloop fermion self-energy corrections are taken into account in the Schwinger-Dyson equations. The propagation properties of the quasiparticles in the dense medium are not the same as in vacuum. A more accurate computation of the gap necessarily needs to take into account this fact. One of the most relevant characteristics of the quasiparticles in the medium is that these have a finite lifetime [13-15]. These quasiparticles scatter with the quarks inside the Fermi sea, and they decay. In all the previous estimates of the color gap these effects have been ignored to leading order. What is the effect of a finite lifetime on the gap equation? Physically, one would expect that the decay of quasiparticles will limit their efficiency to participate in the condensation process.

*Email address: Cristina.Manuel@cern.ch
For those quasiparticles whose lifetime is very short (and this is the case for those who are far away from the Fermi surface), their chance to participate in Cooper pairing is rather small. Taking properly into account the damping rate of the quasiparticles should then allow us to distinguish which are the modes which participate in the condensation. This should then provide a physical ultraviolet cutoff in the gap equation. This is actually the most relevant effect of including the damping rate in the gap equation for BCS superconductivity in weak coupling [16]. Here we want to estimate the lifetime effects in the framework of color superconductivity.

This paper is structured as follows. In Sec. II we write the Schwinger-Dyson equations of the superconducting phase of QCD in the general case when one-loop fermion self-energy corrections are not neglected. We will however neglect vertex corrections throughout our analysis. Those equations are the generalization to color superconductivity of the equations that Eliashberg considered for BCS superconductivity [17]. In the most general case, these equations are very hard to solve. However, in weak coupling the one-loop fermion selfenergies are the same, up to corrections of the order of the square of the condensate, in the superconducting and normal phases of the system. Therefore, one can include the oneloop self-energy corrections of the normal phase of the system in the Schwinger-Dyson equations. With this last approximation, the gap equation can be solved in an easier way. In Sec. III, and for the purposes of comparison, we briefly review the computation of the gap in the free quasiparticle approximation of Ref. [7]. We will only consider the case of $N_{f}=2$ quark massless flavors throughout this article. A generalization of our results to a different number of quark flavors is rather straightforward. In Sec. IV, we derive the gap equation when the one-loop self-energy corrections are included. We give a final expression of the gap equation written in terms of the one-loop spectral density of the quasiparticles at an arbitrary small value of the temperature $T$. To evaluate the lifetime effects at $T=0$, we model this spectral density by a Lorentzian function. We then make a rough estimate of the correction to the value of the gap due to the inclusion of these lifetime effects. Since the damping rate has a linear dependence on the quasiparticle energy [13-15], 
rather than quadratic as in BCS superconductivity, we then find that, to leading order, the effect of the damping rate in the gap is to reduce the effective coupling constant that the quasiparticles close to the Fermi surface see through the condensation process. We present our conclusions in Sec. V.

\section{SCHWINGER-DYSON EQUATIONS IN THE SUPERCONDUCTING PHASE OF QCD AT WEAK COUPLING}

\section{A. Eliashberg equations}

Here we will follow the Nambu-Gorkov (NG) formalism to study the superconducting phase of QCD at very high baryonic density. In the NG basis

$$
\Psi=\left(\begin{array}{c}
\psi \\
\psi_{C}
\end{array}\right), \quad \bar{\Psi}=\left(\bar{\psi}, \bar{\psi}_{C}\right)
$$

where $\psi_{C}(x)=C \bar{\psi}^{T}(x)$ is the charge-conjugate spinor, the Schwinger-Dyson (SD) equations read

$$
S_{\alpha \beta}=S_{\alpha \beta}^{(0)}+S_{\alpha \gamma}^{(0)} \Sigma_{\gamma \delta} S_{\delta \beta},
$$

where the Greek indices refer to the upper and down components of the NG fields. Every term in the NG matrix is also a matrix in color, flavor and Dirac space. We will suppress throughout the color and flavor indices, unless necessary to avoid confusion. The free propagators $S_{\alpha \beta}^{(0)}$ are

$$
S_{11}^{(0)}(P)=\frac{1}{\boldsymbol{P}+\mu \gamma_{0}-m}, \quad S_{22}^{(0)}(P)=\frac{1}{\boldsymbol{P}-\mu \gamma_{0}-m},
$$

while $S_{12}^{(0)}=S_{21}^{(0)}=0$. Here $\Sigma_{\gamma \delta}$ denote the self-energy corrections. Notice that $\Sigma_{21}$ and $\Sigma_{12}$ imply the creation and annihilation, respectively, of a condensate, while the terms $\Sigma_{11}$,
$\Sigma_{22}$ do not. Vertex corrections to the SD equations will be omitted at the order of approximation we are working.

The values of the one-loop propagators can be obtained from the SD equations considered above. After solving the set of coupled equations for $S_{21}$ and $S_{11}$, one finds

$$
S_{21}=-\left(1-S_{22}^{(0)} \Sigma_{22}\right)^{-1} S_{22}^{(0)} \Sigma_{21} S_{11}
$$

and

$$
S_{11}=\left[1-S_{11}^{(0)} \Sigma_{11}-S_{11}^{(0)} \Sigma_{12}\left(1+S_{22}^{(0)} \Sigma_{22}\right)^{-1} S_{22}^{(0)} \Sigma_{21}\right]^{-1} S_{11}^{(0)} .
$$

The gap equation is now derived by demanding the value of the condensate be the same as its one-loop correction. Using the imaginary time formalism, where $K=\left(k_{0}, \mathbf{k}\right)$ and $k_{0}=-i \omega_{n}$, where $\omega_{n}$ is a fermionic Matsubara frequency, ${ }^{1}$

$$
\Sigma_{21}(K)=g^{2} \frac{T}{V} \sum_{Q} \bar{\Gamma}_{a}^{\mu} \Delta_{\mu \nu}^{a b}(K-Q) S_{21}(Q) \Gamma_{b}^{\nu},
$$

where $g$ is the coupling constant, $\Delta_{\mu \nu}^{a b}$ is the gluon propagator, and the vertices are $\Gamma_{a}^{\mu} \equiv T_{a} \gamma^{\mu}$ and $\Gamma_{a}^{\mu} \equiv C\left(\Gamma_{a}^{\mu}\right)^{T} C^{-1} \equiv$ $-\gamma^{\mu} T_{a}^{T}$, and $T_{a}=\lambda_{a} / 2$, where $\lambda_{a}$ are the Gell-Mann matrices.

In a self-consistent treatment of the system, one obtains the fermion self-energy as

$$
\Sigma_{11}(K)=g^{2} \frac{T}{V} \sum_{Q} \Gamma_{a}^{\mu} \Delta_{\mu \nu}^{a b}(K-Q) S_{11}(Q) \Gamma_{b}^{\nu} .
$$

Notice that if one ignores the functions $\Sigma_{11}$ and $\Sigma_{22}$, Eq. (2.6) reduces to the gap equation already considered in the literature.

The gluon propagator of Eqs. (2.6),(2.7) has also to be computed self-consistently. The one-loop gluon self-energy corrections are given by (see $[18,19]$ for an the explicit computation at leading order)

$$
\begin{aligned}
\Pi_{a b}^{\mu \nu}(P)= & \frac{g^{2}}{2} \frac{T}{V} \sum_{K} \operatorname{Tr}_{s, c, f}\left[\Gamma_{a}^{\mu} S_{11}(K) \Gamma_{b}^{\nu} S_{11}(K-P)+\bar{\Gamma}_{a}^{\mu} S_{22}(K) \bar{\Gamma}_{b}^{\nu} S_{22}(K-P)\right. \\
& \left.+\Gamma_{a}^{\mu} S_{12}(K) \bar{\Gamma}_{b}^{\nu} S_{21}(K-P)+\bar{\Gamma}_{a}^{\mu} S_{21}(K) \Gamma_{b}^{\nu} S_{12}(K-P)\right] .
\end{aligned}
$$

\section{B. Weak coupling limit and the Meissner effect}

Equations (2.6)-(2.8) are the generalization of the Eliashberg equations [17] to color superconductivity. They form a set of coupled integral equations, which are extremely hard to solve. In the weak coupling limit, however, this set of equations can be decoupled and simplified, due to the large hierarchy of scales in the theory.

We will first make the approximation that $\Sigma_{11}$ is given, up to corrections of the order of the squared of the condensate, by the value it would take in the normal phase of the system. That is, one can approximate Eq. (2.7) by replacing $S_{11}$ by $S_{11}^{(0)}$
A similar approximation can be made in the gluon propagator. One can use the gluon propagator in Eqs. (2.6),(2.7) with the value it would take in the normal phase of the system, that is, in the hard dense loop (HDL) approximation $[20,21]$, neglecting to leading order the Meissner effect.

The reason why one can neglect the Meissner effect in the computation of $\Sigma_{21}$ was first explain in Ref. [4] and after-

\footnotetext{
${ }^{1}$ Our notation and conventions are almost the same as in Ref. [7], the only change being the notation for the propagators. To compare, just notice that $S_{11 / 22}^{(0)}=G_{0}^{ \pm}$and $\Sigma_{21}=\Phi^{+}, \Sigma_{12}=\Phi^{-}=\gamma_{0}\left(\Phi^{+}\right)^{\dagger} \gamma_{0}$.
} 
wards confirmed in Refs. [5-9]. The scattering processes of quarks close to the Fermi surface, the ones responsible for Cooper's instability, are dominated by small angle (or collinear) scattering. These processes are mediated by the interchange of soft gluons. For soft gluons, the value of the polarization tensor, Eq. (2.8), is dominated by the HDL contribution (see Refs. $[18,19]$ ), while the Meissner effect is subleading.

The reason why one neglects the Meissner effect in the computation of $\Sigma_{11}$ close to the Fermi surface is essentially the same as for $\Sigma_{12}$. The imaginary part of $\Sigma_{11}$ evaluated close to the Fermi surface describes the scattering of a quark close to the Fermi surface with the quarks inside the Fermi sea. This process is dominated by collinear scattering, with the exchange of soft magnetic gluons which have space-like momenta [13-15]. The Landau damping effect of magnetic gluons is then fully dominant with respect to the Meissner effect.

In the approximation of using the HDL gluon propagator in Eqs. (2.6),(2.7), these last integral equations are decoupled from Eq. (2.8). This allows for analytical computations of corrections of order $\mathcal{O}\left(g^{2}\right)$ of the value of the gap, which would be otherwise impossible. Only a numerical analysis, such as the one carried out in Ref. [10], can estimate the corrections introduced by neglecting the Meissner effect. This is beyond the scope of this paper.

\section{GAP EQUATION IN THE FREE QUASIPARTICLE APPROXIMATION}

In this section we review the computation of the gap in the case where we consider that the quasiparticles which form the Cooper pairs are not further affected by medium effects. We will follow closely the computation of Ref. [7], and in the following section we will simply comment on how this computation is modified when the self-energy corrections are included.

From now on, we will only treat the case of two quark flavors in the massless limit. In this case the color, flavor, and Dirac structure of the gap equation simplifies drastically. By restoring color $(i, j, k=1,2,3)$ and flavor $(f, g=1,2)$ indices, $\Sigma_{21}^{i j, f g}=\epsilon_{f g} \epsilon_{i j k} \Phi_{k}^{+}$, and one may take $\Phi_{k}^{+}=\Phi^{+} \delta_{k 3}$. The Dirac structure of the condensate is

$$
\Phi^{+}(K)=\sum_{h=r, l} \sum_{e= \pm} \phi_{h}^{e}(K) \mathcal{P}_{h} \Lambda_{\mathbf{k}}^{e}
$$

where $\mathcal{P}_{h}$, and $\Lambda_{\mathbf{k}}^{e}$ are chirality and energy projectors, respectively:

$$
\mathcal{P}_{r}=\frac{1+\gamma_{5}}{2}, \quad \mathcal{P}_{l}=\frac{1-\gamma_{5}}{2}, \quad \Lambda_{\mathbf{k}}^{ \pm}=\frac{1 \pm \gamma_{0} \gamma \cdot \hat{\mathbf{k}}}{2}
$$

The functions $\phi_{h}^{+}$and $\phi_{h}^{-}$are commonly known as the gap and the antigap, respectively. We will focus on the equation for the gap. The antigap has not even been computed to leading order.

In the presence of the above condensate the color group $S U(3)_{c}$ is broken to $S U(2)_{c}$. The gap equation can be sim- plified if one takes into account that the gauge field modes which contribute the most to the integral are very "soft" [4]. Then, one can take the gluon propagators in the HDL approximation $[20,21]$, neglecting to leading order the Meissner effect. Also, one can drop the chirality index $h$, as the equations for the right- and left-handed gaps at very high density are identical and decoupled. In the Coulomb gauge, the gap equation reduces to [7]

$$
\begin{aligned}
\phi^{+}(K)= & \frac{2}{3} g^{2} \frac{T}{V} \sum_{Q}\left\{\frac{\phi^{+}(Q)}{q_{0}^{2}-(|\mathbf{q}|-\mu)^{2}-\left|\phi^{+}\right|^{2}}\right. \\
& \times\left[\Delta_{L}(K-Q) \frac{1+\hat{\mathbf{k}} \cdot \hat{\mathbf{q}}}{2}+\Delta_{T}(K-Q)\right. \\
& \left.\times\left(-\frac{3-\hat{\mathbf{k}} \cdot \hat{\mathbf{q}}}{2}+\frac{1+\hat{\mathbf{k}} \cdot \hat{\mathbf{q}}}{2} \frac{(k-q)^{2}}{(\mathbf{k}-\mathbf{q})^{2}}\right)\right] \\
& +\frac{\phi^{-}(Q)}{q_{0}^{2}-(|\mathbf{q}|+\mu)^{2}-\left|\phi^{-}\right|^{2}}\left[\Delta_{L}(K-Q) \frac{1+\hat{\mathbf{k}} \cdot \hat{\mathbf{q}}}{2}\right. \\
& \left.\left.+\Delta_{T}(K-Q)\left(-\frac{3-\hat{\mathbf{k}} \cdot \hat{\mathbf{q}}}{2}+\frac{1+\hat{\mathbf{k}} \cdot \hat{\mathbf{q}}}{2} \frac{(k-q)^{2}}{(\mathbf{k}-\mathbf{q})^{2}}\right)\right]\right\},
\end{aligned}
$$

where $\Delta_{L}$ and $\Delta_{T}$ are the longitudinal and transverse HDL propagators, respectively. In the above formula, the gauge dependent pieces of the gluon propagator have been omitted, since, to leading order, they do not contribute in the determination of the gap. Also, the contribution of the quasiantiparticles in the above integral is very much suppressed, and it can be neglected. If one evaluates the gap on shell and very close to the Fermi energy, the integral is dominated by the contribution of very soft Landau-damped magnetic gluons. When the static electric gluons contribution is also taken into account, one then arrives at the integral equation

$$
\phi_{k}=\frac{\bar{g}^{2}}{2} \int_{0}^{\delta} \frac{d(q-\mu)}{\epsilon_{q}}\left[\ln \left(\frac{\mu^{2} b^{2}}{\left|\epsilon_{q}^{2}-\epsilon_{k}^{2}\right|}\right)\right] \phi_{q},
$$

where $\epsilon_{q}=\sqrt{(|\mathbf{q}|-\mu)^{2}+\left|\phi^{+}\right|^{2}}, \quad \phi_{q} \equiv \phi_{q}^{+} \equiv \phi^{+}\left(\epsilon_{q}, \mathbf{q}\right)$, and $\bar{g}=g /(3 \sqrt{2} \pi)$, and

$$
b=256 \pi^{4}\left(\frac{2}{N_{f} g^{2}}\right)^{5 / 2} b_{0}^{\prime},
$$

where $b_{0}^{\prime}$ is a constant of order 1. The integral in Eq. (3.4) is limited to be around the Fermi surface by introducing explicitly a cutoff $\delta \ll \mu$. The final value of the gap finally does not depend on $\delta$. The solution of the above equation for $k \sim \mu$ gives the value of the gap $\phi_{0}$ to leading order in $g$

$$
\phi_{0} \sim 2 \frac{b_{0}}{g^{5}} \mu \exp \left(-\frac{\pi}{2 \bar{g}}\right)[1+\mathcal{O}(\bar{g})]
$$


where $b_{0}=g^{5} b$. In the following sections we will see how lifetime effects of the quasiparticles modify the above result.

\section{GAP EQUATION INCLUDING FERMION SELF-ENERGY CORRECTIONS}

In this section we study how the self-energy corrections to the quark propagators modify the gap equation, when one works in the weak coupling limit. To that end, we first compute in the weak coupling approximation the value of $S_{21}$.

It is very convenient to project all the propagators and self-energy corrections into positive and negative energy contributions. Thus

$$
\begin{aligned}
{\left[S_{11}^{(0)}\right]^{-1}(P)=} & \boldsymbol{P}+\mu \gamma_{0}=\gamma_{0} \Lambda_{\mathbf{p}}^{+}\left(p_{0}+\mu-|\mathbf{p}|\right) \\
& +\gamma_{0} \Lambda_{\mathbf{p}}^{-}\left(p_{0}+\mu+|\mathbf{p}|\right),
\end{aligned}
$$

$$
\begin{aligned}
{\left[S_{22}^{(0)}\right]^{-1}(P)=} & \boldsymbol{P}-\mu \gamma_{0}=\gamma_{0} \Lambda_{\mathbf{p}}^{+}\left(p_{0}-\mu-|\mathbf{p}|\right) \\
& +\gamma_{0} \Lambda_{\mathbf{p}}^{-}\left(p_{0}-\mu+|\mathbf{p}|\right)
\end{aligned}
$$

and $\Sigma_{\alpha \beta}(P)=\Sigma_{e= \pm} \gamma_{0} \Lambda_{\mathbf{p}}^{e} \Sigma_{\alpha \beta}^{e}(P)$, where $\alpha=\beta=1,2$. Notice that in the weak coupling approximation we are considering, both the right- and left-handed quarks get the same one-loop corrections.

We first compute $S_{11}$. We will approximate Eq. (2.5) by

$$
S_{11} \approx\left(1-S_{11}^{(0)} \Sigma_{11}-S_{11}^{(0)} \Sigma_{12} S_{22}^{(0)} \Sigma_{21}\right)^{-1} S_{11}^{(0)}
$$

neglecting one of the quark self-energy corrections which multiplies the condensate. With these approximations, one then arrives at

$$
\begin{aligned}
S_{11}(P)= & \sum_{h=r, l} \mathcal{P}_{h}\left(\Lambda_{\mathbf{p}}^{+} \gamma_{0} \frac{p_{0}-\mu+|\mathbf{p}|}{p_{0}^{2}-(|\mathbf{p}|-\mu)^{2}-\left|\phi_{h}^{+}\right|^{2}-\left(p_{0}-\mu+|\mathbf{p}|\right) \Sigma_{11}^{+}(P)}\right. \\
& \left.+\Lambda_{\mathbf{p}}^{-} \gamma_{0} \frac{p_{0}-\mu-|\mathbf{p}|}{p_{0}^{2}-(|\mathbf{p}|+\mu)^{2}-\left|\phi_{h}^{-}\right|^{2}-\left(p_{0}-\mu-|\mathbf{p}|\right) \Sigma_{11}^{-}(P)}\right)
\end{aligned}
$$

and

$$
\begin{aligned}
S_{21}= & -\sum_{h=r, l} \mathcal{P}_{-h}\left(\frac{\Lambda_{\mathbf{p}}^{-} \phi_{h}^{+}(P)}{\left[p_{0}-\mu+|\mathbf{p}|-\Sigma_{22}^{-}(P)\right]\left(p_{0}+\mu-|\mathbf{p}|-\Sigma_{11}^{+}(P)\right)-\left|\phi_{h}^{+}\right|^{2}}\right. \\
& \left.\times \frac{\Lambda_{\mathbf{p}}^{+} \phi_{h}^{-}(P)}{\left[p_{0}-\mu-|\mathbf{p}|-\Sigma_{22}^{+}(P)\right]\left(p_{0}+\mu+|\mathbf{p}|-\Sigma_{11}^{-}(P)\right)-\left|\phi_{h}^{-}\right|^{2}}\right) .
\end{aligned}
$$

Notice that in Eq. (4.4), we have also neglected terms in the denominators of order

$$
\frac{\Sigma_{22}^{\mp}\left|\phi_{h}^{\mp}\right|^{2}}{p_{0}-\mu \pm|\mathbf{p}|},
$$

which give subleading corrections to the gap equation.

Then, with this fermion propagator, the gap equation reads (we drop the chirality index from now on)

$$
\begin{aligned}
\phi^{+}(K)= & \frac{2}{3} g^{2} \frac{T}{V} \sum_{Q}\left\{\frac { \phi ^ { + } ( Q ) } { [ q _ { 0 } - \mu + | \mathbf { q } | - \Sigma _ { 2 2 } ^ { - } ( Q ) ] [ q _ { 0 } + \mu - | \mathbf { q } | - \Sigma _ { 1 1 } ^ { + } ( Q ) ] - | \phi ^ { + } | ^ { 2 } } \left[\Delta_{L}(K-Q) \frac{1+\hat{\mathbf{k}} \cdot \hat{\mathbf{q}}}{2}\right.\right. \\
& \left.+\Delta_{T}(K-Q)\left(-\frac{3-\hat{\mathbf{k}} \cdot \hat{\mathbf{q}}}{2}+\frac{1+\hat{\mathbf{k}} \cdot \hat{\mathbf{q}}}{2} \frac{(k-q)^{2}}{(\mathbf{k}-\mathbf{q})^{2}}\right)\right]+\frac{\phi^{-}(Q)}{\left[q_{0}-\mu-|\mathbf{q}|-\Sigma_{22}^{+}(Q)\right]\left[q_{0}+\mu+|\mathbf{q}|-\Sigma_{11}^{-}(Q)\right]-\left|\phi^{-}\right|^{2}} \\
& \left.\times\left[\Delta_{L}(K-Q) \frac{1+\hat{\mathbf{k}} \cdot \hat{\mathbf{q}}}{2}+\Delta_{T}(K-Q)\left(-\frac{3-\hat{\mathbf{k}} \cdot \hat{\mathbf{q}}}{2}+\frac{1+\hat{\mathbf{k}} \cdot \hat{\mathbf{q}}}{2} \frac{(k-q)^{2}}{(\mathbf{k}-\mathbf{q})^{2}}\right)\right]\right\} .
\end{aligned}
$$

Taking into account that the relation between the self-energy corrections to the fermion fields and charge-conjugate fields is given by

$$
\Sigma_{22}(K)=-\Sigma_{11}(-K)
$$

so that $\Sigma_{22}^{-}(K)=-\Sigma_{11}^{+}(-K), \quad \Sigma_{22}^{+}(K)=-\Sigma_{11}^{-}(-K)$, we then see that the effect of including self-energy corrections in the gap equation and in the weak coupling limit is to simply modify Eq. (3.3) by making the following replacements: 


$$
\begin{aligned}
& \Xi(Q)^{+}=\frac{\phi^{+}(Q)}{q_{0}^{2}-(|\mathbf{q}|-\mu)^{2}-\left|\phi^{+}\right|^{2}} \rightarrow \Upsilon^{+}(Q)=\frac{\phi^{+}(Q)}{-\left[S_{n}^{+}(-Q)\right]^{-1}\left[S_{n}^{+}(Q)\right]^{-1}-\left|\phi^{+}\right|^{2}}, \\
& \Xi^{-}(Q)=\frac{\phi^{-}(Q)}{q_{0}^{2}-(|\mathbf{q}|+\mu)^{2}-\left|\phi^{-}\right|^{2}} \rightarrow \Upsilon^{-}(Q)=\frac{\phi^{-}(Q)}{-\left[S_{n}^{-}(-Q)\right]^{-1}\left[S_{n}^{-}(Q)\right]^{-1}-\left|\phi^{-}\right|^{2}},
\end{aligned}
$$

where $S_{n}^{+} / S_{n}^{-}$denote the one-loop propagators for quarks and antiquarks, respectively, in the normal phase of the system. The contribution of the quasi-antiparticles in this case is still negligible, and it can be dropped as in the free quasiparticle case.

Let us stress here that after these replacements are done, the propagator $S_{21}$ is a gauge dependent function, as the gluon propagator is in Eq. (2.6). This is so because, in general, $\Sigma_{11}$ and $\Sigma_{22}$ are gauge dependent functions. However, we argue that even after including the one-loop self-energy correction, we will obtain a correction to the gap which is gauge independent, as $\phi_{0}$ in Eq. (3.6) is. The value of $\phi_{0}$ is gauge independent because the main contribution to the integral in Eq. (3.3) arises from almost on-shell quarks which are very close to the Fermi surface [5-7]. In our treatment of the gap equation we will include the medium modifications to these on-shell quarks, and these corrections are also gauge independent [15].

\section{A. Spectral representations}

To perform the sum over Matsubara frequencies in Eq. (4.6) it is convenient to introduce the spectral function representations of both the gauge and fermion propagators. For the gluon propagators we use the same ones as in Ref. [7], that is,

$$
\begin{aligned}
& \Delta_{L}(P) \equiv-\frac{1}{p^{2}}+\int_{0}^{1 / T} d \tau e^{p_{0} \tau} \Delta_{L}(\tau, \mathbf{p}), \\
& \Delta_{T}(P) \equiv \int_{0}^{1 / T} d \tau e^{p_{0} \tau} \Delta_{T}(\tau, \mathbf{p})
\end{aligned}
$$

and

$$
\begin{aligned}
\Delta_{L, T}(\tau, \mathbf{p}) \equiv & \int_{0}^{\infty} d \omega \rho_{L, T}(\omega, \mathbf{q})\left\{\left[1+n_{B}(\omega / T)\right] e^{-\omega \tau}\right. \\
& \left.+n_{B}(\omega / T) e^{\omega \tau}\right\}
\end{aligned}
$$

where $n_{B}(x)=1 /\left(e^{x}-1\right)$, and the spectral functions for the HDL propagators are given in [7]. For the quark propagators,

$$
\Upsilon^{+}(Q) \equiv \int_{0}^{1 / T} d \tau e^{q_{0} \tau} \Upsilon^{+}(\tau, \mathbf{q})
$$

where

$$
\begin{aligned}
\Upsilon^{+}(\tau, \mathbf{q}) \equiv & \int_{0}^{\infty} d \omega \tilde{\rho}_{F}(\omega, \mathbf{q})\left\{\left[1-n_{F}(\omega / T)\right] e^{-\omega \tau}\right. \\
& \left.-n_{F}(\omega / T) e^{\omega \tau}\right\}
\end{aligned}
$$

and $n_{F}(x)=1 /\left(e^{x}+1\right)$.

In the most general case $\tilde{\rho}_{F}(\omega, \mathbf{q})$ will be a non-trivial function of the frequency $\omega$, as opposed to what happens in the free quasiparticle approximation, where it reduces to a delta function

$$
\rho_{F}(\omega, \mathbf{q}) \equiv-\frac{\phi_{q}}{2 \epsilon_{q}} \delta\left(\omega-\epsilon_{q}\right) .
$$

\section{B. Sum over Matsubara frequencies}

When the gluon and fermion propagators are expressed in terms of their spectral densities, one can easily perform the sum over Matsubara frequencies of Eq. (4.6). With $\mathbf{p}=\mathbf{k}-\mathbf{q}$, one finds

$$
\begin{aligned}
T \sum_{q_{0}} \Delta_{L}(K-Q) Y^{+}(Q)= & \int_{0}^{\infty} d \omega^{\prime} \tilde{\rho}_{F}\left(\omega^{\prime}, \mathbf{q}\right)\left\{-\frac{2}{p^{2}} \frac{1}{2} \tanh \left(\frac{\omega^{\prime}}{2 T}\right)\right. \\
& +\int_{0}^{\infty} d \omega \rho_{L}(\omega, \mathbf{p})\left[\frac{1}{2} \tanh \left(\frac{\omega^{\prime}}{2 T}\right)\left(\frac{1}{k_{0}+\omega+\omega^{\prime}}-\frac{1}{k_{0}-\omega-\omega^{\prime}}-\frac{1}{k_{0}-\omega+\omega^{\prime}}+\frac{1}{k_{0}+\omega-\omega^{\prime}}\right)\right. \\
& \left.\left.+\frac{1}{2} \operatorname{coth}\left(\frac{\omega}{2 T}\right)\left(\frac{1}{k_{0}+\omega+\omega^{\prime}}-\frac{1}{k_{0}-\omega-\omega^{\prime}}+\frac{1}{k_{0}-\omega+\omega^{\prime}}-\frac{1}{k_{0}+\omega-\omega^{\prime}}\right)\right]\right\}
\end{aligned}
$$




$$
\begin{aligned}
T \sum_{q_{0}} \Delta_{T}(K-Q) Y^{+}(Q)= & \int_{0}^{\infty} d \omega^{\prime} \tilde{\rho}_{F}\left(\omega^{\prime}, \mathbf{q}\right)\left\{\int _ { 0 } ^ { \infty } d \omega \rho _ { T } ( \omega , \mathbf { p } ) \left[\frac{1}{2} \tanh \left(\frac{\omega^{\prime}}{2 T}\right)\right.\right. \\
& \times\left(\frac{1}{k_{0}+\omega+\omega^{\prime}}-\frac{1}{k_{0}-\omega-\omega^{\prime}}-\frac{1}{k_{0}-\omega+\omega^{\prime}}+\frac{1}{k_{0}+\omega-\omega^{\prime}}\right) \\
& \left.\left.+\frac{1}{2} \operatorname{coth}\left(\frac{\omega}{2 T}\right)\left(\frac{1}{k_{0}+\omega+\omega^{\prime}}-\frac{1}{k_{0}-\omega-\omega^{\prime}}+\frac{1}{k_{0}-\omega+\omega^{\prime}}-\frac{1}{k_{0}+\omega-\omega^{\prime}}\right)\right]\right\}
\end{aligned}
$$

In the most general situation, the frequency integral $\omega^{\prime}$ will be difficult to evaluate analytically, and only a numerical study of the gap equation will be possible. We will concentrate from now on in the zero temperature limit case.

\section{Zero temperature limit}

We evaluate the gap equation at $T=0$ after analytical continuation to Minkowski space is done. We will also make the approximation that, as in Eq. (3.3), the relevant gauge field modes which contribute to the integral are those which are very soft: Landau damped in the magnetic gluon sector and static in the electric gluon one. Thus, one can perform the same type of approximations in the spectral densities of the gluons as the ones done in the free quasiparticle case [7]. Thus, one has to evaluate

$$
\begin{aligned}
\phi_{k}= & \frac{2}{3} g^{2} \int \frac{d^{3} q}{(2 \pi)^{3}} \int_{0}^{\infty} d \omega^{\prime} \tilde{\rho}_{F}\left(\omega^{\prime}, \mathbf{q}\right)\left\{\frac{2}{p^{2}+m_{D}^{2}} \frac{(k+q)^{2}-p^{2}}{4 k p}\right. \\
& +\left[\frac{2 \Theta(p-M)}{p^{2}}+\Theta(M-p)\left(\frac{p^{4}}{p^{6}+M^{4}\left(\epsilon_{k}+\omega^{\prime}\right)^{2}}\right.\right. \\
& \left.\left.\left.+\frac{p^{4}}{p^{6}+M^{4}\left(\epsilon_{k}-\omega^{\prime}\right)^{2}}\right)\right]\left(1+\frac{p^{2}}{4 k q}-\frac{\left(k^{2}-q^{2}\right)^{2}}{4 k q p^{2}}\right)\right\},
\end{aligned}
$$

where $m_{D}^{2}=N_{f} g^{2} \mu^{2} / 2 \pi^{2}$ is the Debye mass, and $M^{2}$ $=(\pi / 4) m_{D}^{2}$.

\section{EVALUATION OF THE LIFETIME EFFECTS IN THE GAP EQUATION}

The frequency integral of Eq. (4.16) thus depends on the spectral density of the fermion propagator. This can only be determined after the computation of $\Sigma_{11}$ is done (see $[12,15])$. As an approximation, we will model this spectral density by a Lorentzian function

$$
\tilde{\rho}_{F}(\omega, \mathbf{q}) \approx-\frac{\phi_{q}}{2 \epsilon_{q}} \frac{Z_{q}}{\pi}\left\{\frac{\Gamma_{q}}{\left(\omega-E_{q}\right)^{2}+\Gamma_{q}^{2}}-\frac{\Gamma_{q}}{\left(\omega+E_{q}\right)^{2}+\Gamma_{q}^{2}}\right\},
$$

where $\Gamma_{q}$ is the damping rate of the quasiparticle. The quasiparticle energies $E_{q}$ should include the effects of the self- energy corrections to the quasiparticle dispersion relations, as they would arise from the real part of $\Sigma_{11}^{+}$. However, since this only displaces by a small amount the poles of the quark propagators, we will ignore this effect, and replace $E_{q}$ by $\epsilon_{q}$. The factor $Z_{q}$ corresponds to the wave function renormalization, and for quasiparticles close to the Fermi surface reads

$$
Z_{q}^{-1}=1-\frac{g^{2}}{9 \pi^{2}} \ln \frac{M}{\epsilon_{q}}
$$

We will approximate this function as $Z_{q} \sim 1$ [4], and simply concentrate in the damping rate effect on the value of the gap.

To evaluate the effects of the damping rate in the gap equation one then only needs to compute the frequency integrals of Eq. (4.16). In the case of pure static interactions, it is easy to check that

$$
\int_{0}^{\infty} d \omega^{\prime} \tilde{\rho}_{F}\left(\omega^{\prime}, \mathbf{q}\right)=-\frac{\phi_{q}}{2 \epsilon_{q}} \frac{2}{\pi} \arctan \left(\frac{\epsilon_{q}}{\Gamma_{q}}\right) .
$$

For non-static magnetic interactions, the result of the integration is more complex. In the limit where we can neglect $\Gamma_{q}$ in front of $\epsilon_{q}$ and $p$ it also reduces to

$$
\begin{aligned}
\int_{0}^{\infty} d \omega^{\prime} \tilde{\rho}_{F}\left(\omega^{\prime}, \mathbf{q}\right)\left(\frac{p^{4}}{p^{6}+M^{4}\left(\epsilon_{k}+\omega^{\prime}\right)^{2}}\right. \\
\left.+\frac{p^{4}}{p^{6}+M^{4}\left(\epsilon_{k}-\omega^{\prime}\right)^{2}}\right) \\
\approx-\left(\frac{p^{4}}{p^{6}+M^{4}\left(\epsilon_{k}+\epsilon_{q}\right)^{2}}\right) \frac{\phi_{q}}{p^{4}} \frac{2}{\pi} \arctan \left(\frac{\epsilon_{q}}{\Gamma_{q}}\right) .
\end{aligned}
$$

After the frequency integral is done, one can treat the angular integrals of Eq. (4.16) using the same approximations as in the free quasiparticle case (see [7]). One then arrives at

$$
\phi_{k}=\frac{\bar{g}^{2}}{2} \int_{0}^{\infty} d(q-\mu)\left[\ln \left(\frac{\mu^{2} b^{2}}{\left|\epsilon_{q}^{2}-\epsilon_{k}^{2}\right|}\right)\right] \frac{\phi_{q}}{\epsilon_{q}} \frac{2}{\pi} \arctan \left(\frac{\epsilon_{q}}{\Gamma_{q}}\right) .
$$


We thus see that, essentially, the effect of the damping rate of the quasiparticles is to modulate the integrand of the gap equation and to introduce a physical cutoff for those cases where the damping rate becomes very large and almost comparable to the energies of the quasiparticles. This modulation is the same as the one that occurs in BCS superconductivity in weak coupling [16]. Nevertheless, a crucial difference arises in the case of color superconductivity; namely, here the damping rate of the quasiparticles depends linearly, rather than quadratically as in BCS, on their energy, when these are close to the Fermi energy.

To get a rough estimate of how the damping rate affects the value of the gap (3.6), we will make the following approximations. The integral is dominated by the contribution of quasiparticles which are close to the Fermi surface. For those quarks ${ }^{2}[13-15]$

$$
\Gamma_{q}=\frac{g^{2} C_{F}}{24 \pi}|| \mathbf{q}|-\mu|+\frac{g^{2} C_{F}}{64 m_{D}}(|\mathbf{q}|-\mu)^{2}+\mathcal{O}\left(\left(\frac{(|\mathbf{q}|-\mu)}{m_{D}}\right)^{3}\right),
$$

where $C_{F}=\left(N^{2}-1\right) / 2 N=\frac{4}{3}$ for quarks in the fundamental representation.

Therefore, in the region where $\Gamma_{q} \ll \epsilon_{q}$ (which holds true for quasiparticles close to the Fermi surface), we will approximate

$$
\frac{2}{\pi} \arctan \left(\frac{\epsilon_{q}}{\Gamma_{q}}\right) \approx 1-\frac{2}{\pi} \frac{\Gamma_{q}}{\epsilon_{q}}
$$

Since for quarks close to the Fermi surface the dominant contribution comes from the linear term in Eq. (5.6), we can approximate

$$
\frac{\Gamma_{q}}{\epsilon_{q}} \approx \frac{g^{2}}{18 \pi} \frac{1}{\sqrt{1+\frac{\left|\phi_{q}\right|^{2}}{(|\mathbf{q}|-\mu)^{2}}}} \approx \frac{g^{2}}{18 \pi}+\mathcal{O}\left(|\phi|^{2}\right) .
$$

Therefore, for the quarks close to the Fermi surface, the effect of the damping rate is to replace Eq. (3.4) by

$$
\phi_{k}=\frac{\bar{g}_{\text {eff }}^{2}}{2} \int_{0}^{\delta} \frac{\delta(q-\mu)}{\epsilon_{q}}\left[\ln \left(\frac{\mu^{2} b^{2}}{\left|\epsilon_{q}^{2}-\epsilon_{k}^{2}\right|}\right)\right] \phi_{q},
$$

where

$$
\bar{g}_{\text {eff }}^{2}=\bar{g}^{2}\left(1-2 \bar{g}^{2}\right)
$$

\footnotetext{
${ }^{2}$ The first term in Eq. (5.6) is due to collinear scattering, that is, by scattering processes with the exchange of soft Landau damped magnetic gluons. These interactions are long ranged, and give a contribution to the damping rate quite different from those of short range interactions (see Ref. [15]). The effects of long range interactions are clearly dominant. This is why the Meissner effect can be neglected to leading order.
}

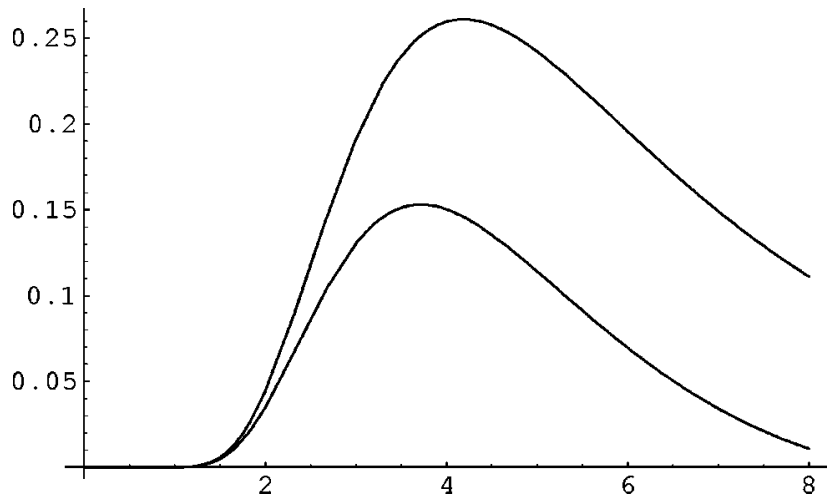

FIG. 1. Plots of $\phi_{0} / \mu$ and $\phi_{0}^{d a m p} / \mu$ as a function of the coupling constant $g$.

The solution to the gap equation can then be obtained simply by replacing $\bar{g}$ in Eq. (3.6) by $\bar{g}_{\text {eff }}$. Therefore, one can conclude that the effect of the damping rate for the quasiparticles close to the Fermi surface is to reduce the effective coupling constant that the quasiparticles see in the condensation process.

To get a much more careful estimate of the lifetime effects, one can alternatively convert the gap equation (5.5) into a differential equation, as done in Refs. [4,6,7]. One then reaches to

$$
\frac{d^{2} \phi(x)}{d x^{2}}=-\bar{g}^{2} \frac{2}{\pi} \arctan \left(\frac{\epsilon(x)}{\Gamma(x)}\right) \phi(x),
$$

where $x=\ln 2 b \mu /\left(k-\mu+\epsilon_{k}\right)$. This equation can only be solved numerically, as $\epsilon$ and $\Gamma$ are complicated functions of $x$. Our approximations to reach to Eq. (5.9) are only valid when $\Gamma \ll \epsilon$ (that is, close to the Fermi surface).

On the contrary, for quasiparticles which are not close to the Fermi surface, their damping rate will be dominated by the higher order terms in the expansion on $\left[(|\mathbf{q}|-\mu) / m_{D}\right]^{n}$ of $\Gamma_{q}$. One thus can state that the Debye mass plays the role of ultraviolet cutoff $\delta$ in the gap equation, as the ratio $\epsilon_{q} / \Gamma_{q}$ starts to be small for $(|\mathbf{q}|-\mu) \gg m_{D}$.

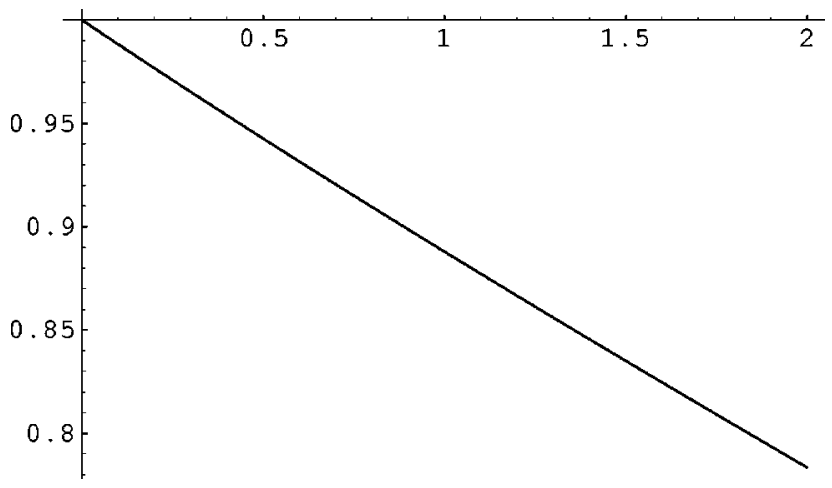

FIG. 2. Ratio of $\phi_{0}^{d a m p} / \phi_{0}$ as a function of the coupling constant $g$. 


\section{CONCLUSIONS}

In this paper we have studied how the one-loop fermion self-energy corrections modify the color gap equation in the weak coupling limit. Then we have focused our study on estimating how the damping rates of the quasiparticles affect the value of the gap, neglecting any other effect, such as, for example, the small displacements of the poles in the quasiparticle one-loop propagators or the wave function renormalization.

The closer to the Fermi surface the quasiparticles are, the longer they live. We have argued that the decay of the quasiparticles limits their efficiency to participate in the Cooper pairing process. A self-consistent inclusion of the damping rate effect in the gap equation also provides the domain in momentum space of the quasiparticles which participate in the condensation. This is also the effect occurring in BCS superconductivity [16].

A rough estimate of how the damping rate of the quasiparticles affects the value of the gap at the Fermi surface gives, to leading order,

$$
\phi_{0}^{\text {damp }} \sim 2 \frac{b_{0}}{g^{5}} \mu \exp \left(-\frac{\pi}{2 \bar{g}_{e f f}}\right),
$$

where $\bar{g}_{\text {eff }}$ is an effective (and reduced) coupling constant given in Eq. (5.10). In principle, the value of $b_{0}$ would also be modified if we had taken into account the wave function renormalization, as stated in Refs. $[11,12]$, but we have neglected those effects in the present article. Better numerical estimates of the damping effects in the value of the gap could be obtained by including the Meissner effect in the gluon propagators of Eqs. (2.6),(2.7). We expect that these effects only modify slightly the leading order behavior obtained in this article.

The value of the gap is then reduced after taking into account the damping rates of the quasiparticles. In the weak coupling limit these effects are small. To get an idea of their relevance we show in Fig. 1 the gaps (3.6) and (6.1) over $\mu$ as a function of the coupling constant, assuming $b_{0}^{\prime}=1$. In Fig. 2 we plot the ratio of $\phi_{0}^{d a m p} / \phi_{0}$ as a function of $g$. For values of $g \sim 0.5$, the reduction of the value of the gap is of the order of $5 \%$. If we could extrapolate the gap to the strong coupling region, as suggested in the literature, the effect would be much more dramatic.

It would be interesting to study how lifetime effects also reduce the critical temperature of transition to the normal phase of the system. To that end, one should first compute the temperature corrections to Eq. (5.6).

\section{ACKNOWLEDGMENTS}

I would like to thank Y. Lozano, M. Tytgat and R. Pisarski for useful discussions.
[1] D. Bailin and A. Love, Phys. Rep. 107, 325 (1984).

[2] M. Alford, K. Rajagopal, and F. Wilczek, Phys. Lett. B 422, 247 (1998).

[3] R. Rapp, T. Schäfer, E. V. Shuryak, and M. Velkovsky, Phys. Rev. Lett. 81, 53 (1998).

[4] D. T. Son, Phys. Rev. D 59, 094019 (1999).

[5] T. Schäfer and F. Wilczek, Phys. Rev. D 60, 114033 (1999).

[6] R. D. Pisarski and D. H. Rischke, Phys. Rev. D 61, 051501 (2000).

[7] R. D. Pisarski and D. H. Rischke, Phys. Rev. D 61, 074017 (2000).

[8] D. K. Hong, Phys. Lett. B 473, 118 (2000).

[9] D. K. Hong, V. A. Miransky, I. A. Shovkovy, and L. C. Wijewardhana, Phys. Rev. D 61, 056001 (2000).

[10] N. Evans, J. Hormuzdiar, S. D. Hsu, and M. Schwetz, Nucl. Phys. B581, 391 (2000).
[11] W. E. Brown, J. T. Liu, and H. Ren, Phys. Rev. D 61, 114012 (2000).

[12] W. E. Brown, J. T. Liu, and H. Ren, Phys. Rev. D 62, 054016 (2000).

[13] M. Le Bellac and C. Manuel, Phys. Rev. D 55, 3215 (1997).

[14] B. Vanderheyden and J. Ollitrault, Phys. Rev. D 56, 5108 (1997).

[15] C. Manuel, Phys. Rev. D 62, 076009 (2000).

[16] P. Morel and P. Nozieres, Phys. Rev. 126, 1909 (1962).

[17] G. M. Eliashberg, Zh. Éksp. Teor. Fiz. 11, 966 (1960) [Sov. Phys. JETP 38, 696 (1960)].

[18] D. H. Rischke, Phys. Rev. D 62, 034007 (2000).

[19] D. H. Rischke, Phys. Rev. D 62, 054017 (2000).

[20] R. D. Pisarski, Phys. Rev. Lett. 63, 1129 (1989); E. Braaten and R. D. Pisarski, Nucl. Phys. B337, 569 (1990).

[21] C. Manuel, Phys. Rev. D 53, 5866 (1996). 